BULL. AUSTRAL. MATH. SOC.

\title{
EVENTUALLY REGULAR SEMIGROUPS
}

\author{
P.M. EDWARDS
}

\begin{abstract}
A semigroup is said to be eventually regular if each of its elements has some power that is regular. Regular and group-bound semigroups are each eventually regular. Idempotent-surjective semigroups are semigroups such that all idempotent congruence classes contain idempotents; eventually regular semigroups are idempotent-surjective. Many results for regular semigroups also hold for eventually regular semigroups or even for idempotentsurjective semigroups and so in particular are also valid for group-bound semigroups. Lallement's lemma is generalized to eventually regular semigroups and the maximum idempotentseparating congruence on such a semigroup is found. Other congruences are considered and the results obtained are applied to yield results on biordered sets.
\end{abstract}

\section{Introduction}

In 1958 Drazin [3] introduced the concept of a pseudo-invertible element of an associative ring or semigroup. He defines a function $x+x^{\prime}$, "somewhat analogous to the generalized inverse function, over the elements of arbitrary finite-dimensional algebras, and even of an extensive class of associative rings" (see [3], p. 506).

Munn [11] studied further these pseudo-inverses in semigroups and found that an element $x$ of a semigroup $S$ is pseudo-invertible if and

Received 17 March 1983. The author thanks his supervisor, Professor G.B. Preston, for his guidance and suggestions throughout the preparation of this paper and also T.E. Hall for his many helpful suggestions. The support of a Commonwealth Postgraduate Research Award is also acknowledged. 
only if some power of $x$ lies in a subgroup of $S$. This naturally leads to the study of group-bound semigroups (see Hall and Munn [7]) which are semigroups in which each element has some power in a subgroup or equivalently, semigroups in which all elements are pseudo-invertible.

Not all regular semigroups are group-bound. Define a semigroup to be eventually regular if each of its elements has some power that is regular. Thus in particular if a semigroup is regular or group-bound it is an eventually regular semigroup.

A generalization of the concept of eventually regular will also prove. convenient. Define a semigroup $S$ to be idempotent-surjective if whenever $\rho$ is a congruence on $S$ and $a \rho$ is an idempotent congruence class in $S / \rho$, then ap contains an idempotent of $S$. It will be shown that all eventually regular semigroups are idempotent-surjective semigroups.

In Section 3 similarities between regular, eventually regular and idempotent-surjective semigroups are explored and it is shown that many results for regular semigroups extend to eventually regular semigroups or even to idempotent-surjective semigroups. Thus in particular these results are also valid for group-bound semigroups.

Lal lement's lemma ([9], Lemma 2.2) is extended to eventually regular semigroups. In fact a stronger result will be shown namely that if $S$ is eventually regular and $\phi$ is a morphism from $S$ onto a semigroup $T$ such that, for $c$ and $d$ in $S, c \phi$ and $d \phi$ are mutual inverses in $T$, then there exist $a, b$ in $S$ such that $a \phi=c \phi$ and $b \phi=d \phi$ with $a$ and $b$ mutual inverses in $S$.

We define a relation, denoted throughout this paper by $\mu$, on an arbitrary semigroup $S$ and show that it is an idempotent-separating congruence. For an eventually regular semigroup $S$, we show that $\mu$ is the maximum idempotent-separating congruence on $S$ and also show (see Example 3 in Section 5) that a semigroup may have a maximum idempotentseparating congruence that is distinct from $\mu$.

In Section 4, some of the results obtained in Section 3 are applied to biordered sets and conditions are found on a congruence $\rho$ on a semigroup $S$, such that the biordered set $E(S / \rho)$ is isomorphic to the biordered set $E(S)$. It is shown that $S / \mu$ is finite if and only if $E(S)$ is finite and it is further shown that if $S$ is an idempotent-surjective 
semigroup then $E(S / \mu) \cong E(S)$.

Section 5 contains some counterexamples to possible extensions of the results to arbitrary semigroups.

\section{Preliminaries}

Whenever possible the notations and conventions of $\mathrm{Cl}$ ifford and Preston [1, 2] or Howie [8] are used. Further for any semigroup $S$ define a relation $V=V(S)$ (as in Hall [6]) by

$$
V=\{(a, b) \in S \times S \mid a b a=a \text { and } b a b=b\},
$$

and for each $a$ in $S$, denote $\{b \in S \mid(a, b) \in V\}$ by $V(a)$.

A semigroup $S$ is called eventually regular if for each element $a$ in $S$ there is a positive integer $n$ (in general depending on $a$ ) such that $a^{n}$ is regular.

If $T$ is a semigroup then the set of idempotents of $T$ will be denoted by $E(T)$. The set $E(T)$ will be regarded where necessary, as equipped with an appropriate structure, such as that of a biordered set, as will be clear from the context.

A congruence on a semigroup is called idempotent-surjective if every idempotent congruence class contains an idempotent and idempotent-creative otherwise. Thus a congruence $\rho$ on a semigroup $S$ is idempotentsurjective if and only if the natural map $\rho^{\xi}$ restricted to $E(S)$ is a surjection of $E(S)$ upon $E(S / \rho)$. A semigroup is called idempotentsurjective if all of its congruences are idempotent-surjective.

The empty set will be denoted by $\square$, the identity relation on a set $X$ will be denoted by $I_{X}$ and the conjunction of $L_{a} \leq L_{b}$ and $R_{a} \leq R_{b}$ will be denoted by $H_{a} \leq H_{b}$.

\section{Eventualiy regular semigroups}

In this section the relationship between regular, group-bound, eventually regular and idempotent-surjective semigroups is investigated. A regular or group-bound semigroup is clearly eventually regular. By extending a version of Lallement's lemma ([9], Lemma 2.2) to eventually regular semigroups it is shown that all eventually regular semigroups are 
idempotent-surjective semigroups.

In preparation for the next section, this section concludes with an investigation of idempotent-separating congruences on eventually regular semigroups including the determination of the maximum such congruence.

THEOREM 1. Let $S$ be an eventually regular semigroup and let $\phi$ be a morphism of $S$ onto a semigroup $T$ with $(c, d) \in V(T)$. Then for any $x, y$ in $S$ such that $x \phi=c$ and $y \phi=d$ there exist elements $a, b, r$, $s$ in $S$ such that $a=x r x, b=y s y, a \phi=c, b \phi=d$ and $(a, b) \in V(S)$.

Proof. As $\phi$ is onto $T$ there exist $x, y$ in $S$ such that $x \phi=c$ and $y \phi=d$. As $S$ is eventually regular there exists an integer $n \geq 1$ such that $\left((x y)^{2}\right)^{n}$ is regular. Let $z \in V\left((x y)^{2 n}\right)$ and put

$a=(x y)^{2 n-1} z x y x$ and $b=y(x y)^{2 n-2} z x y$ (if $n=1$ put $b=y z x y$ ). It is easy to verify that $(a, b) \in V(S)$.

As $z \in V\left((x y)^{2 n}\right), x \phi=c, y \phi=d$ and $\phi$ is a morphism it follows that $z \phi \in V\left((c d)^{2 n}\right)$. As $(c, d) \in V(T),(c d)^{2 n}=c d$. Thus $z \phi \in V(c d)$ and so will be denoted $(c d)^{\prime}$. As $\phi$ is a morphism,

$$
\begin{array}{rlrl}
a \phi & =(c d)^{2 n-1}(c d)^{\prime} c d c & \\
& =c d(c d)^{\prime} c d c, & & \text { as }(c, d) \in V(T), \\
& =c d c, & & \text { as }(c d)^{\prime} \in V(c d), \\
& =c & &
\end{array}
$$

and, if $n>1$,

$$
\begin{aligned}
b \phi & =d(c d)^{2 n-2}(c d)^{\prime} c d & & \\
& =d(c d)^{\prime} c d, & & \text { as }(c, d) \in V(T), \\
& =d c d(c d)^{\prime} c d, & & \text { as } d=d c d, \\
& =d c d, & & \text { as }(c d)^{\prime} \in V(c d), \\
& =d . & &
\end{aligned}
$$

If $n=1$ then $b \phi=d(c d)^{\prime} c d=d$ as above. It can now be seen that $a$ and $b$ meet all of the requirements of the theorem.

In our terminology Lallement's lemma states that regular semigroups are idempotent-surjective. As a corollary of Theorem 1, the following generalization of Lallement's lemma holds. 
COROLLARY 2. Eventually regular semigroups are idempotent-surjective semigroups. Indeed if $S$ is an eventually regular semigroup, $\rho$ is a congmence on $S$, and $w \rho$ is an idempotent of $S / \rho$ then an idempotent $e$ can be found in wo such that $H_{e} \leq H_{w}$.

Proof. Let $w \rho=(w \rho)^{2}$ in $S / \rho$. Then $(w \rho, w \rho) \in V(S / \rho)$ and so by Theorem 1 (using $x=y=w$ ) there exist $a, b, r, s$ in $s$ such that $a=w r w, b=w s w, a \rho=w \rho=b \rho$ and $(a, b) \in V(S)$. Putting $e=a b$ yields $e=e^{2}, e=w r u w s w$ and $e \rho=(a b) \rho=a \rho b \rho=w \rho w \rho=w \rho$. Thus $e$ is an idempotent in $w \rho$ and $H_{e} \leq H_{w}$ as required.

When applied to regular semigroups Theorem 1 reduces to Lemma 1 of Hall [6]. For group-bound semigroups, in particular for finite semigroups, Theorem 1 appears to be new.

THEOREM 3. Let $S$ be con eventually regular semigroup and $\rho$ a congruence on $S$ contained in $L$. Then for each element $a$ in $S, a$ is an element of a subgroup of $S$ if and only if $a \rho$ is an element of a subgroup of $S / \rho$.

Proof. Just use Theorem 1 instead of Hall's Lemma 1 in his proof of Theorem 15 in [6].

THEOREM 4. Let $S$ be an idempotent-surjective semigroup with $E(S) \neq \square$. Define a relation $\sigma$ on $S$ by

$$
\sigma=\left\{(e f, f e) \in S \times S \mid e^{2}=e, f^{2}=f\right\} .
$$

Let $\sigma^{*}$ be the congruence generated by $\sigma$. Then $E\left(S / \sigma^{*}\right)$ is a semilattice and so the set of regular elements of $S / \sigma^{*}$ is an inverse semgroup. Also $\sigma^{*}$ is the finest congruence that has this property and moreover, if $\rho$ is a congruence on $S$ with $\sigma^{*} \subseteq \rho$, then the regular elements of $s / \rho$ form an inverse semigroup.

Proof. As $S$ is idempotent-surjective each idempotent congruence class contains an idempotent. This together with the definition of $\sigma$ * easily leads to a proof of the claims made in the theorem.

A corollary to this theorem is Result 3 of Hall [5], namely that if $S$ is regular then $\sigma^{*}$ is the finest inverse congruence on $S$.

For the definition of categorical at zero and its related concepts see 
Clifford and Preston ([2], 73-80). The following result of Hall will be used.

RESULT A ([4], Theorem 1). Let $S=S^{0}$ be a semigroup which is categorical at zero. Define

$$
\rho_{1}=\left\{(e, f) \in S \times S \mid e=e^{2}, f=f^{2}, e f=f e=f \neq\right. \text { 아. }
$$

Let $\rho_{1}^{*}$ be the congmence generated by $\rho_{1}$. Define by induction $\rho_{m+1}^{*}$, the congmence generated by

$$
\rho_{m+1}=\left\{(e, f) \in S \times S \mid e \rho_{m}^{*} e^{2}, f \rho_{m}^{*} f^{2},(e f) \rho_{m}^{*}(f e) \rho_{m}^{*} f \neq 0\right\} .
$$

Define $\rho=\bigcup_{n=1}^{\infty} \rho_{n}^{*}$. Then $\rho$ is the finest o-restricted primitive congmence on $S$. (Note $\rho_{1}^{*}$ is a 0-restricted congruence.)

LEMMA 5. Let $S=S^{0}$ be a semigroup with the following properties:

(a) $S$ is categorical at zero;

(b) $S$ is idempotent-surjective;

(c) each of the (Zocal) subsemigroups eSe, $e=e^{2}$ is idempotent-surjective.

Let $\rho$ and $\rho_{1}$ be defined as in Result A. Then $\rho=\rho_{1}^{*}$.

Proof. It suffices to show that $S / \rho_{1}^{*}$ is primitive since $\rho_{1}^{*} \subseteq \rho$ $\left(S / \rho_{1}^{*}\right.$ primitive implies that $\rho \subseteq \rho_{1}^{*}$ by Result A). Suppose $A=A^{2}$, $B=B^{2}$ and $0 \neq A \leq B$ in $S / \rho_{1}^{*}$. Then since $S$ is idempotent-surjective there exists $b=b^{2} \in B$ and because $B A B=A=A^{2}, A \in E\left(B\left(S / \rho_{1}^{*}\right) B\right)$. Since $b S b$ is idempotent-surjective, $\left.\left(\rho_{1}^{*}\right)^{h}\right|_{E(b S b)}$ maps $E(b S b)$ onto $E\left(B\left(S / \rho_{1}^{*}\right) B\right)$. Therefore, since $A \in E\left(B\left(S / \rho_{1}^{*}\right) B\right)$, there exists $a \in E(b S b)$ such that $(a)\left(\rho_{1}^{*}\right)^{4}=A$. From $a \in E(b S b)$ it follows that $a=a^{2}$ and $a \leq b$. Note that $a \in A$ and $a \neq 0$ since $A \neq 0$ in $S / \rho_{1}^{*}$. Thus 
$0 \neq a \leq b$, and so from the definition of $\rho_{1}$ we have $a \rho_{1} b$, whence $A=B$ and so $S / \rho_{1}^{*}$ is primitive.

THEOREM 6. If $S=S^{0}$ is an eventually regular semigroup which is categorical at zero and $\rho$ and $\rho_{1}$ are defined as in Result $A$ then $\rho=\rho_{1}^{*}$.

Proof. If $S$ is an eventually regular semigroup and $e=e^{2} \epsilon S$ then the subsemigroup $e S e$ is also eventually regular because if $a \in e S e$, with $a^{n}$ regular in $S$ and $x \in V\left(a^{n}\right)$, then exe $\in V\left(a^{n}\right) n e S e$. Therefore both $S$ and eSe are idempotent-surjective by Corollary 2 and so the result follows from Lemma 5 .

This generalizes the first assertion of Theorem 2 of [4] from regular to eventually regular semigroups.

LEMMA 7. Let $\sigma$ be an idempotent-separating congmence on an eventually regular semigroup $S$ and let $(e, b) \in \sigma$ with $e=e^{2}$. Then $H_{e} \leq H_{b}$.

Proof. Since $b \sigma$ is an idempotent congruence class and $S$ is eventually regular, by Corollary 2 there exists an idempotent $f$ in bo such that $H_{f} \leq H_{b}$. However since $\sigma$ is idempotent-separating and $(e, f) \in \sigma$ it follows that $e=f$, whence $H_{e} \leq H_{b}$.

DEFINITION. For any semigroup $S$ define a relation $\mu$ by $\mu=\{(a, b) \in S \times S \mid$ if $x \in S$ is regular then each of $x$ Rea, $x$ Rxb implies $x a H x b$, and each of $x L a x, x L b x$ implies $a x f b x\}$.

THEOREM 8. For any semigroup $S$ the relation $\mu$ is an idempotentseparating congruence.

Proof. It is clear that the relation $\mu$ is both reflexive and symmetric. Suppose $(a, b) \in \mu$ and $(b, c) \in \mu$. Let $x$ be regular and let $x R_{x a}$. From the definition of $\mu$, we then have $x a+H x b$. Since $H \subseteq R$ it follows that $x R x b$, whence again from the definition of $\mu$, $x b H x c$. Hence saH $x c$. Similarly, $x$ Lax with $x$ regular implies axticx. Thus by the symmetry of the conditions on $a$ and $c,(a, c) \in \mu$ 
and so $\mu$ is transitive.

Take $(a, b) \in \mu$ and $y \in S$. Let $x R x(a y)$ with $x$ regular. In any semigroup $x$ Rxay implies $x R x a$ and so it follows from the definition of $\mu$ that $x a+h x b$. As $x a y R_{x a}$ there exists $u$ in $S^{l}$ such that xayu $=x a$. As $x a H x b$ there exists $v \in S^{l}$ such that $x b=v x a$. Thus $(x b y) u=(v x a) y u=v(x a b u)=v x a=x b$ and so $x b R x b y$, whence $x b y R_{x b} R_{x a} R_{x} R_{x a y}$. Since $x a H x b$, it follows that $x a L_{x b} b$; hence xaylxby, since $L$ is a right congruence. Thus $x(a y) H x(b y)$.

Now let $x L(a y) x$ with $x$ regular. Then $x$ Lyx and so $y x$ is regular. Thus $y x$ Layx with $y x$ regular from which it follows that $a(y x) H b(y x)$, since $(a, b) \in \mu$. Hence $(a y) x H(b y) x$. By symmetry of the conditions on $a$ and $b$ it follows that $(a y, b y) \in \mu$. Dually, $(y a, y b) \in \mu$ and so $\mu$ is compatible.

To see that the congruence $\mu$ is idempotent-separating consider $(e, f) \in \mu$ with $e^{2}=e, f^{2}=f$. Then by putting $x=e$ in the definition for $\mu$, eRee implies ethef, and similarly $f$ Lff implies efHf. Thus eHf and so $e=f$.

That $\mu$ is a congruence may also be seen by checking that $\mu=\operatorname{ker} \phi$ where $\phi$ is the following representation of $S$ (verbal communication from T.E. Hall). Let $X$ be the set of regular L-classes of $S$ and $Y$ be the set of regular R-classes of $S$. Then define $\phi: S \rightarrow P T_{X} \times P T_{Y}^{*}$ by $s \phi=\left(\rho_{s}, \lambda_{s}\right)$, where $\rho_{s}: L_{x} \rightarrow L_{x s}$ if $x$ is regular and $x \operatorname{Rxs}$, and is undefined otherwise, and $\lambda_{s}: R_{x} \rightarrow R_{s x}$ if $x$ is regular and $x$ Lsx, and is undefined otherwise. (Note $P T_{Y}^{*}$ denotes the dual of the semigroup $P T_{Y} \cdot$ )

THEOREM 9. The following are equivalent for a congruence $\sigma$ on a semigroup $S$ :

(i) $\sigma \subseteq \mu$;

(ii) for all $e \in E(S)$, for all $b$ in $S,(e, b) \in \sigma$ implies $H_{e} \leq H_{b}$;

(iii) for all regular elements $a$ in $S$, for all $b$ in $S$, 


$$
(a, b) \in \sigma \text { implies } H_{a} \leq H_{b}
$$

and if $S$ is eventually regular then each of the above is equivalent to

(iv) $\sigma$ is idempotent-separating.

Proof. That (i) implies ( $i$ ) follows easily from the definition of $\mu$.

We now show that (ii) implies (iii). Assume (ii) and let $(a, b) \in \sigma$ with $a$ regular. Take $a^{\prime} \in V(a)$. Then since $\left(a a^{\prime}, b a^{\prime}\right) \in \sigma$ and $a a^{\prime} \in E(S)$ by $(i i), H_{a a^{\prime}} \leq H_{b a^{\prime}}$. Therefore $R_{a}=R_{a a^{\prime}} \leq R_{b a^{\prime}} \leq R_{b}$ and a dual argument to the above yields $L_{a} \leq L_{b}$, whence $H_{a} \leq H_{b}$.

We now show that (iii) implies (i). Assume (iii) and take $(a, b) \in \sigma$. Let $x R x a$ with $x$ regular. Then as $(x a, x b) \in \sigma$ with $x a$ regular by (iii), $H_{x a} \leq H_{x b}$. Therefore $R_{x}=R_{x a a} \leq R_{x b} \leq R_{x}$, whence $x R x b$ and $x b$ is regular. Therefore, by (iii) again, $H_{x b} \leq H_{x a}$ and so $H_{x a}=H_{x b}$. A dual argument shows $x$ Lax implies $a x t b x$. These implications together with symmetry show that $(a, b) \in \mu$, whence $\sigma \subseteq \mu$.

The equivalence of $(i),(i i)$ and $(i i i)$ is now clear and that (i) implies ( $i v)$ is trivial. If $S$ is eventually regular then (iv) implies (ii) by Lemma 7 . Thus if $S$ is eventually regular then all four conditions are equivalent. (The equivalence of $(i$ ) and (ii) has also been noted by D. Easdown in an unpublished note.)

COROLLARY 10 . Let $R$ denote the set of regular elements of an arbitrary semigroup $S$ and denote the maximum congmence contained in $H$ on $S$ by $H^{b}$. Then

$$
\begin{aligned}
& \text { (a) } H^{b} \subseteq \mu, \\
& \text { (b) if } a, b \in R \text { and }(a, b) \in \mu \text { then atlb, } \\
& \text { (c) } H^{b} \cap(R \times R) \subseteq \mu \cap(R \times R) \subseteq H \cap(R \times R) \text {, } \\
& \text { (d) if } S \text { is regular then } \mu=H^{b} \text {. }
\end{aligned}
$$

Proof. (a) is clear; (b) follows from the equivalence of ( $i$ ) and (iii) in Theoren 9; (c) follows from $(a)$ and (b); and (d) follows easily 
from $(c)$

THEOREM 11. If $S$ is an eventually regular semigroup then $\mu$ is the maximu idenpotent-separating congruence on $S$.

Proof. The proof follows immediately from Theorem 9.

Note that, as Example 3 in Section 5 shows, there exist semigroups which have a maximum idempotent-separating congruence not equal to $\mu$.

\section{Biordered sets}

Let $\rho$ be a congruence on a semigroup $S$. The results of the previous section can in some cases be used to determine when the idempotents of $S$ and of $S / \rho$ form isomorphic biordered sets. We also show that, if $S$ is an eventually regular semigroup, then its biordered set of idempotents is an M-biordered set (for definition, see below).

First, some background material on biordered sets will be useful. For a definition of a biordered set, its related axioms and concepts see Nambooripad [12] or Meakin [10]. The notation of [12] and [10] will be used and is as follows.

Let $S$ be a semigroup and $E \neq \square$ be its set of idempotents. Define $\omega^{2}=\{(e, f) \in E \times E \mid e f=e\}, \omega^{r}=\{(e, f) \in E \times E \mid f e=e\}$, $\omega=\omega^{2} n \omega^{r}, \quad k=\omega^{2} \cup \omega^{r}, L=\omega^{2} n\left(\omega^{2}\right)^{-1}, \quad R=\omega^{r} n\left(\omega^{r}\right)^{-1}$ and $D_{E}=\kappa \cup \kappa^{-1}$. Also for $e \in E$ and $\gamma=\omega^{2}$ or $\omega^{r}$, put $\gamma(e)=\{f \in E \mid f \gamma e\}$. Then the partial binary algebra $E$ with domain $D_{E}$ (that is, the product $e f$ is defined in $E$ if and only if $(e, f) \in D_{E}$ ) is a biordered set ([12], Theorem 1.1). A mapping $\theta: E \rightarrow E^{\prime}$ is a morphism (called bimorphism by Nambooripad and Meakin) of the biordered set $E$ into the biordered set $E^{\prime}$ if $(e, f) \in D_{E}$ implies $(e \theta, f \theta) \in D_{E^{\prime}}$ and $(e f) \theta=(e \theta)(f \theta)$. The biordered sets $E$ and $E^{\prime}$ are isomorphic (denoted by $E \cong E^{\prime}$ ) if there exists a bijection $\theta: E \rightarrow E^{\prime}$ such that both $\theta$ and $\theta^{-1}$ are morphisms.

DEFINITIONS. Let $E$ be a biordered set. For $e, f \in E$, define $M(e, f)=\omega^{l}(e) \cap \omega^{r}(f)$. Define $E$ to be an $M$-biordered set if 
$M(e, f) \neq \square$ for all $e, f \in E$ and a semigroup $S$ to be an $M$-semigroup if $E(S)$ is an $M$-biordered set. Define along the lines of Meakin [10], an arbitrary biordered set $E$ to be rectangular if for all $e, f \in E$ there exists an element $g$ in $E$ such that $e R g$ and $g L f$. Then Meakin's Proposition 1.3 of [10] can be modified to give

RESULT B. For an arbitrary biordered set $E$ the following are equivalent:

(a) $E$ is a rectanguzar biordered set;

(b) $E$ is an M-biondered set and $\omega=I_{E}$;

(c) $E$ is the biordered set of some rectangular band.

LEMMA 12. Let $\sigma$ be an idempotent-surjective congmence contained in $\mu$ on a semigroup $S$. Suppose $A, B \in S / \sigma$ with $A=A^{2}$ and $B=B^{2}$. Then

(i) if $A B=B$ in $S / \sigma$, there exist $a=a^{2} \in A$ and $b=b^{2} \in B$ and, for any such idempotents $a$ and $b$, the equation $a b=b$ holds in $S$; and

(ii) if $A B=A$ in $S / \sigma$, there exist $a=a^{2} \in A$ and $b=b^{2} \in B$ and, for any such idempotents $a$ and $b$, the equation $a b=a$ holds in $S$.

Proof. Since $\sigma$ is an idempotent-surjective congruence there exist $a=a^{2} \in A$ and $b=b^{2} \in B$. Let $A B=B$. Then $(a b) \sigma=b \sigma$. Thus $(a b, b) \in \sigma$, whence since $\sigma \subseteq \mu,(a b, b) \in \mu$. By taking $x=b$ in the definition of $\mu$, from $b L b b$, we have $b b H a b b$, that is, $b$ Hab , from which it follows easily that $a b=b$. Similarly, if $A B=A$, then for $a$ and $b$ as before, $a b=a$.

LEMMA 13. Let $\sigma$ be an idempotent-surjective congruence contained in $\mu$ on a semigroup $S$. Then $E(S) \cong E(S / \sigma)$; furthermore $\left.\sigma^{h}\right|_{E(S)}$ is such an isomorphism.

Proof. If $E(S)=\square$ there is nothing to prove. Otherwise, since $\sigma$ is an idempotent-surjective congruence which is idempotent-separating 
(since $\sigma \subseteq \mu$ ), $\left.\sigma\right|_{E(S)}=\alpha$, say, is a bijection from $E(S)$ upon $E(S / \sigma)$. By Lemma 12, $\alpha^{-1}$ preserves the biordered set relations of $E(S / \sigma)$; that, conversely $\alpha$ preserves the biordered set relations of $E(S)$ is clear. Therefore both $\alpha$ and $\alpha^{-1}$ are morphisms and so $E(S) \cong E(S / \sigma)$.

COROLLARY 14. Let $\sigma$ be an idempotent-separating congruence on an eventually regular semigroup $S$. Then $E(S / \sigma) \cong E(S)$ and so in particular $E(S / \mu) \cong E(S)$.

Proof. By Theorem 1l, $\sigma \subseteq \mu$, and by Corollary 2, $\sigma$ is idempotentsurjective. Thus, by Lemma $13, E(S / \sigma) \cong E(S)$.

THEOREM 15. Let $S$ be any semigroup. Then $S / \mu$ is finite if and only if $E(S)$ is finite. Furthermore, if $\mu$ is an idempotent-surjective congruence, then $E(S / \mu) \cong E(S)$.

Proof. If $E(S)$ is finite then there are only finitely many distinct regular $L$ or $R$-classes. Thus the sets $X$ and $Y$ mentioned in Theorem 8 are finite and the morphism $\phi$ of Theorem 8 has a finite image. Since $\mu=\operatorname{ker} \phi$, it follows that $S / \mu$ is finite. The converse follows from Theorem 8 and the latter assertion is immediate from Lemma 13.

COROLLARY 16. Let $S$ be an eventually regular semigroup. Then $E(S) \cong E(T)$, for some finite semigroup $T$, if and only if $E(S)$ is finite.

Proof. If $E(S)$ is finite the result follows from Corollary 14 and Theorem 15 by taking $T=S / \mu$. The converse is immediate.

Another observation obtainable from Theorem 15 is that the class of biordered sets arising from finite semigroups coincides with the class of biordered sets arising from semigroups with only finitely many idempotents and for which $\mu$ is an idempotent-surjective congruence. Example 4 of Section 5 is of a finite biordered set that comes from no finite semigroup and Example 3 is of a semigroup for which $\mu$ is not an idempotentsurjective congruence and $E(S / \mu) \nsubseteq E(S)$.

The sufficient conditions mentioned in the previous few results are in some cases necessary as shown by the next theorem. 
THEOREM 17. Let $S$ be con eventually regular semigroup with $E(S)$ finite. Then for any congmence $\rho$ on $S, E(S / \rho) \cong E(S)$ if and only if $\rho \subseteq \mu$.

Proof. If $\rho$ is not contained in $\mu$ then by Theorem $11, \rho$ is not idempotent-separating. This in conjunction with Corollary 2 yields $|E(S / \rho)|<|E(S)|$. Therefore $E(S / \rho) \neq E(S)$. The converse follows from Corollary 14 .

The requirement that $E(S)$ be finite is clearly needed since, for example, any infinite chain $C$ has $\mu={ }^{1} C$ and has non-trivial congruences $\rho$ such that $C \cong C / \rho$. Theorem 17 also has the following immediate corollary.

COROLLARY 18. If $\rho$ is a congruence on a finite semigroup $S$ then $E(S / \rho) \cong E(S)$ if and only if $\rho \subseteq \mu$.

PROPOSITION 19. Any eventually regular semigroup is an M-semigroup.

Proof. Let $S$ be an eventually regular semigroup (so $E(S) \neq \square$ ). Let $e, f$ be elements of $E(S)$; then there exists $n \geq 1$ such that $\left((f e)^{2}\right)^{n}$ is regular. Take $x \in V\left((f e)^{2 n}\right)$ and put $k=(f e)^{n} x(f e)^{n}$. Then $k^{2}=k, k e=k$ and $f k=k$ so $k \in M(e, f)$.

Example 4 , below, shows that there is an $M$-semigroup whose biordered set is not the biordered set of any eventually regular semigroup. Thus the class of $M$-biordered sets is more extensive than the class of biordered sets arising from eventually regular semigroups. From Proposition 19 , a necessary condition for a finite biordered set $E$ to come from a finite semigroup is that $E$ be an $M$-biordered set; but again Example 4 shows that this is not sufficient.

\section{Examples}

Not all eventually regular semigroups are regular or group-bound as shown by Example 1 .

EXAMPLE 1 . Let $T$ and $R$ be eventually regular semigroups and $S=T \times R$ be the direct product of $T$ and $R$. It is easy to verify that if $T$ is regular or group-bound then $S$ is eventually regular. Thus if $T$ is a group-bound semigroup that is not regular (for example let $T$ be 
the two element null semigroup) and if $R$ is a regular semigroup that is not group-bound (for example, let $R$ be the bicyclic semigroup) then $S$ is an eventually regular semigroup that is neither regular nor group-bound.

Many of the results obtained in the two previous sections are not true for arbitrary semigroups as shown by the following three examples.

EXAMPLE 2. As a nontrivial example of the ideas developed in the previous sections let $S$ be the semigroup given by Table $1 . S$ is not regular but $S$ is eventually regular since $S$ is finite $\left(e, f, 0, a^{2}\right.$ are regular\}. The $\mu$-classes are $\{e\},\{f\}$, and $\{0, a\}$. Note that each idempotent $\mu$-class contains an idempotent, that is, $\mu$ is an idempotentsurjective congruence (cf. Corollary 2).

$$
E(S)=\{e, f, 0\}, E(S / \mu)=\{e \mu, f \mu, 0 \mu\} \text { and } E(S) \cong E(S / \mu)
$$

(cf. Theorem 15).

Note that $S$ eventually regular, $(x, y) \in \mu$ with $x$ regular does not imply that $y$ is also regular as can be seen from $(0, a) \epsilon \mu$ above.

TABLE 1

\begin{tabular}{l|llll} 
& $e$ & $f$ & $a$ & 0 \\
\hline$e$ & $e$ & $a$ & $a$ & 0 \\
$f$ & 0 & $f$ & 0 & 0 \\
$a$ & 0 & $a$ & 0 & 0 \\
0 & 0 & 0 & 0 & 0
\end{tabular}

EXAMPLE 3. Let $F$ be a free semigroup on a set $X$. Let $S$ be the semigroup constructed from $F$ by adjoining two idempotent elements $e, 1$ such that $e w=w e=1 w=w l=w$ for all $w$ in $F$ and $e l=1 e=e$. Then $S$ is not eventually regular. For this semigroup $S, \mu$ has as its congruence classes $F,\{e\}$ and $\{1\}$. Note that $F$ is an idempotent element of $S / \mu$ but contains no idempotent of $S$ (cf. Corollary 2).

$S$ has another idempotent-separating congruence $\rho$ with congruence classes $F \cup e$ and $\{1\}$. Thus $\mu$ is strictly contained in $\rho$ which is in fact the maximum idempotent-separating congruence on $S$ ( $c f$. Theorem 11).

Moreover $E(S)=\{e, 1\}$ with ewl. $E(S / \mu)=\{F,\{e\},\{1\}\}$ with 
Ew $\{e\} \omega\{1\}$ so certainly $E(S) \neq E(S / \mu)$. Thus the second assertion of Corollary 14 is not true for arbitrary semigroups. The congruence $\rho$ is idempotent-surjective and shows that both the latter assertion in Corollary 2 and Lemma 7 are false for idempotent-surjective congruence classes of arbitrary semigroups.

Finally the congruence $\sigma$ on $S$ whose congruence classes are $\{1, e\} \sigma$ and $F$ is not idempotent-separating but $E(S / \sigma)=\{F,\{e, 1\}\}$ with $F w\{1\}$ and so $E(S / \sigma) \cong E(S)$. In this case there was both a collapse and a creation of idempotents which in effect mutually cancelled each other.

EXAMPLE 4 (Hall). Let $R$ be the free semigroup on the idempotent generators $e, f$. Extend $R$ to a semigroup $P$ by adjoining idempotents $g, h$ satisfying $g r=r g=g, h r=r h=h$ for all $r \in R$ and with $g R h$. Let $E$ be the biordered set of $P$. Then $E=\{e, f, g, h\}$, for which the relations gwe, gwf, hwe, hwf and $g R h$ hold, is an $M$-biordered set and so $P$ is an $M$-semigroup. However $E$ is not the biordered set of an eventually regular semigroup.

Proof. If $E=E(S)$ with $S$ eventually regular then, as in the proof of Proposition 19, there exists $k=(f e)^{n} x(f e)^{n} \in M(e, f)$ with $x \in V\left((f e)^{2 n}\right) . M(e, f)=\{g, h\}$ so without loss of generality $k=g$ (by symmetry). As $g=(f e)^{n} x(f e)^{n}$ and gwe and gwf it follows that $g=(f e)^{n}(f e)^{n} x(f e)^{n}(f e)^{n}=(f e)^{2 n}$. From $g=(f e)^{2 n}$ and hwe and hwf it follows that $h=h(f e)^{2 n}=h g$, whereas, from $h R g$ we have $h g=g$, a contradiction.

\section{References}

[1] A.H. Clifford and G.B. Preston, The algebraic theory of semigroups, Volume I (Mathematical Surveys, 7. American Mathematical Society, Providence, Rhode Island, 1961).

[2] A.H. Clifford and G.B. Preston, The algebraic theory of semigroups, Volume II (Mathematical Surveys, 7. American Mathematical Society, Providence, Rhode Island, 1967). 
[3] M.P. Drazin, "Pseudo-inverses in associative rings and semigroups", Amer. Math. Monthly 65 (1958), 506-514.

[4] T.E. Hall, "Primitive homomorphic images of semigroups", J. Austral. Math. Soc. 8 (1968), 350-354.

[5] T.E. Hall, "On regular semigroups whose idempotents form a subsemigroup", Bull. Austral. Math. Soc. 1 (1969), 195-208.

[6] T.E. Hall, "Congruences and Green's relations on regular semigroups", GLasgow Math. J. 13 (1972), 167-175.

[7] T.E. Hall and W.D. Munn, "Semigroups satisfying minimal conditions II", GLasgow Math. J. 20 (1979), 133-140.

[8] J.M. Howie, An introduction to semigroup theory (London Mathematical Society Monographs, 7. Academic Press, London, New York, San Francisco, 1976).

[9] Gérard Lallement, "Congruences et équivalences de Green sur un demigroupe régulier", C.R. Acad. Sci. Paris Sér. A 262 (1966), 613-616.

[10] John Meakin, "Constructing biordered sets", Semigroups, 67-84 (Proc. Conf. Monash Univ., Clayton, 1979. Academic Press, New York, 1980).

[11] W.D. Munn, "Pseudo-inverses in semigroups", Proc. Cambridge Philos. Soc. 57 (1961), 247-250.

[12] K.S.S. Nambooripad, Structure of regular semigroups. I (Memoirs of the American Mathematical Society, 22. American Mathematical Society, Providence, Rhode Island, 1979).

Department of Mathematics,

Monash University,

Clayton,

Victoria 3168 ,

Australia. 